\title{
SOME INTEGRAL INEQUALITIES OF THE VOLTERRA TYPE
}

\author{
CHYNG NAN CHOU AND GOU-SHENG YANG
}

\begin{abstract}
In this paper we establish some integral inequalities of Volterra type which provide explicit bounds on unknown functions. These inequalities can be used as convenient tools in the study of certain integral equations.
\end{abstract}

\section{Introduction}

The inequalities which provide explicit bounds on unknown functions play a fundamental role in the development of the theory of differential, integral and finite difference equations, see $[1,3,4]$ and the references given therein. In [5] B. G. Pachpatte proved the following interesting theorem:

Theorem A. Let $U(t) \in C\left(R_{+}, R_{+}\right), K(t, s), D_{1} K(t, s) \in C\left(D, R_{+}\right)$for $(t, s) \in D=$ $\left\{(t, \sigma) \in R_{+}^{2}: 0 \leq \sigma \leq t<\infty\right\}$ and $C \geq 0$ is a constant

$\left(\mathrm{a}_{1}\right)$ If

$$
U^{2}(t) \leq C+\int_{0}^{t} K(t, \sigma) U(\sigma) d \sigma
$$

for $t \in R_{+}$, then

$$
U(t) \leq \sqrt{C}+\frac{1}{2} \int_{0}^{t} A(s) d s
$$

for $t \in R_{+}$, where

$$
A(t)=K(t, t)+\int_{0}^{t} D_{1} K(t, \sigma) d \sigma, \quad t \in R_{+} .
$$

$\left(\mathrm{a}_{2}\right)$ Let $g(U)$ be a continuous, nondecreasing function defined on $R_{+}$and $g(U)>0$ on $(0, \infty)$. If

$$
U^{2}(t) \leq C+\int_{0}^{t} K(t, \sigma) U(\sigma) g(U(\sigma)) d \sigma
$$

Received April 12, 2004.

2000 Mathematics Subject Classification. 26D15.

Key words and phrases. Explicit bounds, Volterra type, integral inequalities, differential equations. 
for $t \in R^{+}$, then for $0 \leq t \leq t_{1}, t, t_{1} \in R_{+}$,

$$
U(t) \leq G^{-1}\left[G(\sqrt{C})+\frac{1}{2} \int_{0}^{t} A(s) d s\right], \text { where } G(r)=\int_{r_{0}}^{r} \frac{d s}{g(s)}, \quad r>0, \quad r_{0}>0
$$

$G^{-1}$ is the inverse of $G$ and $t_{1} \in R_{+}$is chosen so that

$$
G(\sqrt{C})+\frac{1}{2} \int_{0}^{t} A(s) d s \in \operatorname{Dom}\left(G^{-1}\right)
$$

for all $t \in R_{+}$lying in the interval $0 \leq t \leq t_{1}$.

Theorem B. Let $u(x, y) \in C\left(R_{+}^{2}, R_{+}\right), p(x, y, s, t), D_{1} p(x, y, s, t), D_{2} p(x$, $y, s, t), D_{2} D_{1} p(x, y, s, t) \in C\left(E, R_{+}\right)$for $(x, y, s, t) \in E=\left\{(x, y, s, t) \in R_{+}^{4}: 0 \leq s \leq\right.$ $x<\infty, 0 \leq t \leq y<\infty\}$ and $C \geq 0$ is a constant.

$\left(\mathrm{b}_{1}\right)$ If

$$
u^{2}(x, y) \leq C+\int_{0}^{x} \int_{0}^{y} p(x, y, \sigma, w) u(\sigma, w) d w d \sigma
$$

for $x, y \in R_{+}$, then

$$
u(x, y) \leq \sqrt{C}+\frac{1}{2} \int_{0}^{x} \int_{0}^{y} B(s, t) d t d s
$$

for $x, y \in R_{+}$, where

$$
\begin{aligned}
B(x, y)= & p(x, y, x, y)+\int_{0}^{x} D_{1} p(x, y, \sigma, y) d \sigma+\int_{0}^{y} D_{2} p(x, y, x, w) d w \\
& +\int_{0}^{x} \int_{0}^{y} D_{2} D_{1} p(x, y, \sigma, w) d w d \sigma, \quad x, y \in R_{+} .
\end{aligned}
$$

$\left(\mathrm{b}_{2}\right)$ Let $g(u)$ be as in $\left(a_{2}\right)$. If

$$
u^{2}(x, y) \leq C+\int_{0}^{x} \int_{0}^{y} p(x, y, \sigma, w) u(\sigma, w) g(u(\sigma, w)) d w d \sigma,
$$

for $x, y \in R_{+}$, then for $0 \leq x \leq x_{1}, 0 \leq y \leq y_{1}, x, x_{1}, y, y_{1} \in R_{+}$,

$$
u(x, y) \leq G^{-1}\left[G(\sqrt{C})+\frac{1}{2} \int_{0}^{x} \int_{0}^{y} B(s, t) d t d s\right]
$$

where $B(x, y)$ is as defined in $\left(b_{1}\right), G, G^{-1}$ are as defined in $\left(a_{2}\right)$ and $x_{1}, y_{1} \in R_{+}$ are chosen so that

$$
G(\sqrt{C})+\frac{1}{2} \int_{0}^{x} \int_{0}^{y} B(s, t) d t d s \in \operatorname{Dom}\left(\mathrm{G}^{-1}\right),
$$

for all $x, y \in R_{+}$lying in $0 \leq x \leq x_{1}, 0 \leq y \leq y_{1}$. 
The main purpose of the present paper is to offer some results dealing with variants of the above inequalities which can be used to study the qualitative behavior of solutions of certain nonlinear integral equations, and some applications are also given to illustrate the usefulness.

\section{Main Results}

In what follows, $R$ denotes the set of real numbers and $R_{+}=[0, \infty)$. We denote by $D=\left\{(t, \sigma) \in R_{+}^{2}: 0 \leq \sigma \leq t<\infty\right\}, E=\left\{(x, y, s, t) \in R_{+}^{4}: 0 \leq s \leq x<\infty, 0 \leq t \leq\right.$ $y<\infty\}$, and $C(M, N)$ the class of continuous functions from the set $M$ to the set $N$. For function $Z(x, y), x, y \in R_{+}$, denote $D_{1} Z(x, y)=\frac{\partial}{\partial x} Z(x, y), D_{2} Z(x, y)=\frac{\partial}{\partial y} Z(x, y)$, $D_{2} D_{1} Z(x, y)=\frac{\partial^{2}}{\partial y \partial x} Z(x, y)$. We assume that all the integrals involved throughout the discussion exist on the respective domains of their definitions.

Theorem 1. Let $U(t), f(t), f^{\prime}(t) \in C\left(R_{+}, R_{+}\right), K(t, s), D_{1} K(t, s) \in C\left(D, R_{+}\right)$. (c) If

$$
U^{2}(t) \leq f(t)+\int_{0}^{t} K(t, \sigma) U(\sigma) d \sigma
$$

for $t \in R_{+}$, then

$$
U(t) \leq \sqrt{f(t)}+\frac{1}{2} \int_{0}^{t} A(s) d s
$$

for $t \in R_{+}$, where

$$
A(t)=K(t, t)+\int_{0}^{t} D_{1} K(t, \sigma) d \sigma
$$

$t \in R^{+}$.

$\left(\mathrm{c}_{2}\right)$ Let $g(u)$ be a continuous, nondecreasing function defined on $R_{+}$and $g(U)>0$ on $(0, \infty)$. If

$$
U^{2}(t) \leq f(t)+\int_{0}^{t} K(t, \sigma) U(\sigma) g(U(\sigma)) d \sigma,
$$

then for $0 \leq t \leq t_{1}, t, t_{1} \in R_{+}$

$$
U(t) \leq G^{-1}\left[G(\sqrt{f(t)})+\frac{1}{2} \int_{0}^{t} A(s) d s\right],
$$

where $A(t)$ is defined by (1.3),

$$
G(r)=\int_{r_{0}}^{r} \frac{d s}{g(s)}, \quad r>0, r_{0}>0 .
$$

$G^{-1}$ is the inverse of $G$ and $t_{1} \in R_{+}$is chosen so that $G(\sqrt{f(t)})+\frac{1}{2} \int_{0}^{t} A(s) d s$ $\in \operatorname{Dom}\left(G^{-1}\right)$, for all $t \in R_{+}$lying in the interval $0 \leq t \leq t_{1}$. 
Proof $\left(\mathbf{c}_{1}\right)$. First we assume that $f(t)>0$ for $t \in R_{+}$. Define a function $Z(t)$ by the right side of (1.1). Then $Z(0)=f(0), U(t) \leq \sqrt{Z(t)}, Z(t)$ is positive and nondecreasing for $t \in R_{+}$and

$$
\begin{aligned}
Z^{\prime}(t) & =f^{\prime}(t)+K(t, t) U(t)+\int_{0}^{t} D_{1} K(t, \sigma) U(\sigma) d \sigma \\
& \leq f^{\prime}(t)+K(t, t) \sqrt{Z(t)}+\int_{0}^{t} D_{1} K(t, \sigma) \sqrt{Z(\sigma)} d \sigma \\
& \leq f^{\prime}(t)+K(t, t) \sqrt{Z(t)}+\int_{0}^{t} D_{1} K(t, \sigma) \sqrt{Z(t)} d \sigma \\
& =f^{\prime}(t)+A(t) \sqrt{Z(t)} \quad t \in R_{+} .
\end{aligned}
$$

From (1.7) and the hypotheses on $K$ and $U$, we observe that

$$
\begin{aligned}
\sqrt{Z(t)} & =\sqrt{Z(0)}+\int_{0}^{t} \frac{1}{2} \frac{Z^{\prime}(s)}{\sqrt{Z(s)}} d s \\
& \leq \sqrt{f(0)}+\int_{0}^{t} \frac{1}{2} \cdot \frac{f^{\prime}(s)+A(s) \sqrt{Z(s)}}{\sqrt{Z(s)}} d s \\
& =\sqrt{f(0)}+\int_{0}^{t} \frac{f^{\prime}(s)}{2 \sqrt{Z(s)}} d s+\frac{1}{2} \cdot \int_{0}^{t} A(s) d s \\
& \leq \sqrt{f(0)}+\int_{0}^{t} \frac{f^{\prime}(s)}{2 \sqrt{f(s)}} d s+\frac{1}{2} \int_{0}^{t} A(s) d s \\
& =\sqrt{f(0)}+(\sqrt{f(t)}-\sqrt{f(0)})+\frac{1}{2} \int_{0}^{t} A(s) d s \\
& =\sqrt{f(t)}+\frac{1}{2} \int_{0}^{t} A(s) d s
\end{aligned}
$$

Using (1.8) in $U(t) \leq \sqrt{Z(t)}$, we get the desired inequality in (1.2).

If $f(t)$ is nonnegative, we carry out the above procedure with $f(t)+\varepsilon$ instead of $f(t)$, where $\epsilon>0$ is an arbitrary small constant, and subsequently pass to the limit as $\epsilon \rightarrow 0$ to obtain (1.2).

$\left(c_{2}\right)$ Let $f(t)>0$ for $t \in R_{+}$and define a function $W(t)$ by the right side of (1.4). Then $W(0)=f(0), U(t) \leq \sqrt{W(t)}, W(t)$ is positive and nondecreasing for $t \in R_{+}$. As in the proof of (1.7), we have

$$
W^{\prime}(t) \leq f^{\prime}(t)+A(t) \sqrt{W(t)} g(\sqrt{W(t)})
$$

From (1.9) and the hypotheses on $K, U$ and $g$, it is easy to observe that

$$
G(\sqrt{W(t)})-G(\sqrt{W(0)})=\int_{0}^{t} \frac{1}{g(\sqrt{W(s)})} \frac{W^{\prime}(s)}{2 \sqrt{W(s)}} d s
$$




$$
\begin{aligned}
& \leq \frac{1}{2} \int_{0}^{t} \frac{f^{\prime}(s)}{g(\sqrt{W(s)}) \sqrt{W(s)}} d s+\frac{1}{2} \int_{0}^{t} A(s) d s \\
& \leq \frac{1}{2} \int_{0}^{t} \frac{f^{\prime}(s)}{g(\sqrt{f(s)}) \sqrt{f(s)}} d s+\frac{1}{2} \int_{0}^{t} A(s) d s \\
& =G(\sqrt{f(t)})-G(\sqrt{f(0)})+\frac{1}{2} \int_{0}^{t} A(s) d s \\
& =G(\sqrt{f(t)})-G(\sqrt{W(0)})+\frac{1}{2} \int_{0}^{t} A(s) d s
\end{aligned}
$$

which implies

$$
G(\sqrt{W(t)}) \leq G(\sqrt{f(t)})+\frac{1}{2} \int_{0}^{t} A(s) d s .
$$

From (1.10) and the hypothesis on $G$ we observe that

$$
\sqrt{W(t)} \leq G^{-1}\left[G(\sqrt{f(t)})+\frac{1}{2} \int_{0}^{t} A(s) d s\right]
$$

Using (1.11) and $U(t) \leq \sqrt{W(t)}$, we get the required inequality in (1.5).

The proof of the case when $f(t) \geq 0$ can be completed as mentioned in the proof of part $\left(c_{1}\right)$.

Remark 1. We note that Theorem A is a special case of Theorem 1.

We next establish a two independent variable version of Theorem 1 which can be used in certain applications.

Theorem 2. Let $U(x, y), f(x, y), D_{1} f(x, y), D_{2} f(x, y) \in C\left(R_{+}^{2}, R_{+}\right), P(x, y, s, t)$, $D_{1} P(x, y, s, t), D_{2} P(x, y, s, t), D_{2} D_{1} P(x, y, s, t) \in C\left(E, R_{+}\right)$.

$\left(\mathrm{d}_{1}\right)$ If

$$
U^{2}(x, y) \leq f(x, y)+\int_{0}^{x} \int_{0}^{y} P(x, y, \sigma, w) U(\sigma, w) d w d s,
$$

for $x, y \in R_{+}$, then

$$
U(x, y) \leq \sqrt{f(x, y)}+\frac{1}{2} \int_{0}^{x} \int_{0}^{y} B(s, t) d t d s
$$

for $x, y \in R_{+}$, where

$$
\begin{aligned}
B(x, y)= & P(x, y, x, y)+\int_{0}^{x} D_{1} P(x, y, \sigma, y) d \sigma+\int_{0}^{y} D_{2} P(x, y, x, w) d w \\
& +\int_{0}^{x} \int_{0}^{y} D_{2} D_{1} P(x, y, \sigma, w) d w d \sigma
\end{aligned}
$$

$x, y \in R_{+}$. 
$\left(\mathrm{d}_{2}\right)$ Let $g(U)$ be a differentiable nondecreasing function defined on $R_{+}$and $g(U)>0$ on $(0, \infty)$. If

$$
U^{2}(x, y) \leq f(x, y)+\int_{0}^{x} \int_{0}^{y} P(x, y, \sigma, w) U(\sigma, w) g(U(\sigma, w)) d w d \sigma
$$

for $x, y \in R_{+}$, then for $0 \leq x \leq x_{1}, 0 \leq y \leq y_{1}, x, x_{1}, y, y_{1} \in R_{+}$,

$$
U(x, y) \leq G^{-1}\left[G(\sqrt{f(x, y)})+\frac{1}{2} \int_{0}^{x} \int_{0}^{y} B(s, t) d t d s\right],
$$

where $B(x, y)$ is defined by $(2.3), G, G^{-1}$ are as defined in $\left(a_{2}\right)$ and $x_{1}, y_{1} \in R_{+}$are chosen so that

$$
G(\sqrt{f(x, y)})+\frac{1}{2} \int_{0}^{x} \int_{0}^{y} B(s, t) d t d s \in \operatorname{Dom}\left(G^{-1}\right)
$$

for all $x, y \in R_{+}$lying in $0 \leq x \leq x_{1}, 0 \leq y \leq y_{1}$.

Proof $\left(\mathbf{d}_{1}\right)$. It is sufficient to assume that $f(x, y)>0$ for $(x, y) \in R_{+}^{2}$, since the standard limiting argument can be used to treat the remaining case. Define a function $Z(x, y)$ by the right hand side of (2.1). Then $Z(0, y)=f(0, y), Z(x, 0)=f(x, 0)$, $U(x, y) \leq \sqrt{Z(x, y)}, Z(x, y)$ is positive and nondecreasing in $x, y \in R_{+}$and

$$
\begin{aligned}
D_{1} Z(x, y)= & D_{1} f(x, y)+\int_{0}^{y} P(x, y, x, w) U(x, w) d w \\
& +\int_{0}^{x} \int_{0}^{y} D_{1} P(x, y, \sigma, w) U(\sigma, w) d w d \sigma \\
D_{2} Z(x, y)= & D_{2} f(x, y)+\int_{0}^{x} P(x, y, \sigma, y) U(\sigma, y) d \sigma \\
& +\int_{0}^{x} \int_{0}^{y} D_{2} P(x, y, \sigma, w) U(\sigma, w) d w d \sigma \\
D_{2} D_{1} Z(x, y)= & D_{2} D_{1} f(x, y)+P(x, y, x, y) U(x, y) \\
& +\int_{0}^{y} D_{2} P(x, y, x, w) U(x, w) d w+\int_{0}^{x} D_{1} P(x, y, \sigma, y) U(\sigma, y) d \sigma \\
& +\int_{0}^{x} \int_{0}^{y} D_{2} D_{1} P(x, y, \sigma, w) U(\sigma, w) d w d \sigma \\
\leq & D_{2} D_{1} f(x, y)+\left[P(x, y, x, y)+\int_{0}^{y} D_{2} P(x, y, x, w) d w\right. \\
& \left.+\int_{0}^{x} D_{1} P(x, y, \sigma, y) d \sigma+\int_{0}^{x} \int_{0}^{y} D_{2} D_{1} P(x, y, \sigma, w) d w d \sigma\right] \sqrt{Z(x, y)} \\
= & D_{2} D_{1} f(x, y)+B(x, y) \sqrt{Z(x, y)}
\end{aligned}
$$

From (2.6), $D_{1} Z(s, t) \geq D_{1} f(s, t)$ and the hypotheses on $f, P$ and $U$ we observe that

$$
\sqrt{Z(x, y)}-\sqrt{Z(0, y)}
$$




$$
\begin{aligned}
& =\int_{0}^{x} \frac{1}{2} \frac{D_{1} Z(s, y)}{\sqrt{Z(s, y)}} d s \\
& =\int_{0}^{x}\left[\frac{D_{1} Z(s, y)}{2 \sqrt{Z(s, y)}}-\frac{D_{1} Z(s, 0)}{2 \sqrt{Z(s, 0)}}\right] d s+\int_{0}^{x} D_{1} \sqrt{Z(s, 0)} d s \\
& =\int_{0}^{x} \int_{0}^{y} D_{2}\left(\frac{D_{1} Z(s, t)}{2 \sqrt{Z(s, t)}}\right) d t d s+\int_{0}^{x} D_{1} \sqrt{Z(s, 0)} d s \\
& \leq \frac{1}{2} \int_{0}^{x} \int_{0}^{y}\left(\frac{D_{2} D_{1} f(s, t)}{\sqrt{Z(s, t)}}+B(s, t)\right) d t d s-\frac{1}{4} \int_{0}^{x} \int_{0}^{y} \frac{D_{1} Z(s, t) D_{2} Z(s, t)}{(\sqrt{Z(s, t)})^{3}} d t d s \\
& +\int_{0}^{x} D_{1} \sqrt{Z(s, 0)} d s \\
& =\frac{1}{2} \int_{0}^{x}\left[\left.\frac{D_{1} f(s, t)}{\sqrt{Z(s, t)}}\right|_{0} ^{y}-\int_{0}^{y}\left(-\frac{1}{2}\right) \frac{D_{1} f(s, t) D_{2} Z(s, t)}{(\sqrt{Z(s, t)})^{3}} d t\right] d s+\frac{1}{2} \int_{0}^{x} \int_{0}^{y} B(s, t) d t d s \\
& -\frac{1}{4} \int_{0}^{x} \int_{0}^{y} \frac{D_{1} Z(s, t) D_{2} Z(s, t)}{(\sqrt{Z(s, t)})^{3}} d t d s+\int_{0}^{x} D_{1} \sqrt{Z(s, 0)} d s \\
& =\left[\frac{1}{2} \int_{0}^{x} \frac{D_{1} f(s, y)}{\sqrt{Z(s, y)}} d s-\frac{1}{2} \int_{0}^{x} \frac{D_{1} f(s, 0)}{\sqrt{Z(s, 0)}} d s\right]+\frac{1}{2} \int_{0}^{x} \int_{0}^{y} B(s, t) d t d s \\
& -\frac{1}{4} \int_{0}^{x} \int_{0}^{y} \frac{D_{2} Z(s, t)}{(\sqrt{Z(s, t)})^{3}}\left(D_{1} Z(s, t)-D_{1} f(s, t)\right) d t d s+\int_{0}^{x} D_{1} \sqrt{Z(s, 0)} d s \\
& \leq \frac{1}{2} \int_{0}^{x} \frac{D_{1} f(s, y)}{\sqrt{Z(s, y)}} d s-\int_{0}^{x} D_{1} \sqrt{Z(s, 0)} d s+\frac{1}{2} \int_{0}^{x} \int_{0}^{y} B(s, t) d t d s+\int_{0}^{x} D_{1} \sqrt{Z(s, 0)} d s \\
& \leq \frac{1}{2} \int_{0}^{x} \frac{D_{1} f(s, y)}{\sqrt{f(s, y)}} d s+\frac{1}{2} \int_{0}^{x} \int_{0}^{y} B(s, t) d t d s \\
& =\sqrt{f(x, y)}-\sqrt{f(0, y)}+\frac{1}{2} \int_{0}^{x} \int_{0}^{y} B(s, t) d t d s \\
& =\sqrt{f(x, y)}-\sqrt{Z(0, y)}+\frac{1}{2} \int_{0}^{x} \int_{0}^{y} B(s, t) d t d s
\end{aligned}
$$

which implies

$$
\sqrt{Z(x, y)} \leq \sqrt{f(x, y)}+\frac{1}{2} \int_{0}^{x} \int_{0}^{y} B(s, t) d t d s
$$

Using $(2.7)$ in $U(x, y) \leq \sqrt{Z(x, y)}$ we get the required inepuality in (2.2).

$\left(\mathrm{d}_{2}\right)$ Let $f(x, y)>0$ for $(x, y) \in R_{+}^{2}$ and define a function $W(x, y)$ by the right hand side of (2.4). Then $W(0, y)=f(0, y), W(x, 0)=f(x, 0), U(x, y) \leq \sqrt{W(x, y)}, W(x, y)$ 
is positive and nondecreasing in $x, y \in R_{+}$and

$$
\begin{aligned}
D_{1} W(x, y)= & D_{1} f(x, y)+\int_{0}^{y} P(x, y, x, w) U(x, w) g(U(x, w)) d w \\
& +\int_{0}^{x} \int_{0}^{y} D_{1} P(x, y, \sigma, w) U(\sigma, w) g(U(\sigma, w)) d \sigma d w \\
D_{2} W(x, y)= & D_{2} f(x, y)+\int_{0}^{x} P(x, y, \sigma, w) U(\sigma, w) g(U(\sigma, w)) d \sigma \\
& +\int_{0}^{x} \int_{0}^{y} D_{2} P(x, y, \sigma, w) U(\sigma, w) g(U(\sigma, w)) d \sigma d w \\
D_{2} D_{1} W(x, y)= & D_{2} D_{1} f(x, y)+P(x, y, x, y) U(x, y) g(U(x, y)) \\
& +\int_{0}^{y} D_{2} P(x, y, x, w) U(x, w) g(U(x, w)) d w \\
& +\int_{0}^{x} D_{1} P(x, y, \sigma, y) U(\sigma, y) g(U(\sigma, y)) d \sigma \\
& +\int_{0}^{x} \int_{0}^{y} D_{2} D_{1} P(x, y, \sigma, w) U(\sigma, w) g(U(\sigma, w)) d \sigma d w \\
\leq & D_{2} D_{1} f(x, y)+\left[P(x, y, x, y)+\int_{0}^{y} D_{2} P(x, y, x, w) d w\right. \\
& +\int_{0}^{x} D_{1} P(x, y, \sigma, y) d \sigma \\
& \left.+\int_{0}^{x} \int_{0}^{y} D_{2} D_{1} P(x, y, \sigma, w) d \sigma d w\right] \sqrt{W(x, y)} g(\sqrt{W(x, y)}) \\
\leq & D_{2} D_{1} f(x, y)+B(x, y) \sqrt{W(x, y)} g(\sqrt{W(x, y)}) . \\
&
\end{aligned}
$$

From (2.8) and the hypotheses on $P, U, g$ and $G$, it is easy to observe that

$$
\begin{aligned}
& G(\sqrt{W(x, y)})-G(\sqrt{W(0, y)})=\int_{0}^{x} \frac{1}{g(\sqrt{W(s, y)})} \frac{1}{2} \frac{D_{1} W(s, y)}{\sqrt{W(s, y)}} d s \\
= & \int_{0}^{x}\left\{\left[\frac{D_{1} W(s, y)}{g(\sqrt{W(s, y)}) 2 \sqrt{W(s, y)}}-\frac{D_{1} W(s, 0)}{g(\sqrt{W(s, 0)}) 2 \sqrt{W(s, 0)}}\right]\right. \\
& \left.+\frac{D_{1} W(s, 0)}{g(\sqrt{W(s, 0)}) 2 \sqrt{W(s, 0)}}\right\} d s \\
= & \frac{1}{2} \int_{0}^{x} \int_{0}^{y} D_{2}\left(\frac{D_{1} W(s, t)}{g(\sqrt{W(s, t)}) \sqrt{W(s, t)}}\right) d t d s+\int_{0}^{x} D_{1} G(\sqrt{W(s, 0)}) d s \\
= & \frac{1}{2} \int_{0}^{x} \int_{0}^{y} \frac{1}{g^{2}(\sqrt{W(s, t)}) W(s, t)}\left\{g(\sqrt{W(s, t)}) \sqrt{W(s, t)} D_{2} D_{1} W(s, t)\right.
\end{aligned}
$$




$$
\begin{aligned}
& \left.-D_{1} W(s, t)\left[g^{\prime}(\sqrt{W(s, t)}) \frac{D_{2} W(s, t)}{2 \sqrt{W(s, t)}} \sqrt{W(s, t)}+g(\sqrt{W(s, t)}) \frac{1}{2} \frac{D_{2} W(s, t)}{\sqrt{W(s, t)}}\right]\right\} d t d s \\
& +\int_{0}^{x} D_{1} G(\sqrt{W(s, 0)}) d s \\
& =\frac{1}{2} \int_{0}^{x} \int_{0}^{y} \frac{D_{2} D_{1} W(s, t)}{g(\sqrt{W(s, t)}) \sqrt{W(s, t)}} d t d s \\
& -\frac{1}{4} \int_{0}^{x} \int_{0}^{y} \frac{g^{\prime}(\sqrt{W(s, t)}) \sqrt{W(s, t)}+g(\sqrt{W(s, t)})}{g^{2}(\sqrt{W(s, t)})(W(s, t))^{3 / 2}} D_{1} W(s, t) D_{2} W(s, t) d t d s \\
& +\int_{0}^{x} D_{1} G(\sqrt{W(s, 0)}) d s \\
& \leq \frac{1}{2} \int_{0}^{x} \int_{0}^{y} \frac{D_{2} D_{1} f(s, t)}{g(\sqrt{W(s, t)}) \sqrt{W(s, t)}} d t d s+\frac{1}{2} \int_{0}^{x} \int_{0}^{y} B(s, t) d t d s \\
& -\frac{1}{4} \int_{0}^{x} \int_{0}^{y} \frac{g^{\prime}(\sqrt{W(s, t)}) \sqrt{W(s, t)}+g(\sqrt{W(s, t)})}{g^{2}(\sqrt{W(s, t)})(W(s, t))^{3 / 2}} D_{1} W(s, t) D_{2} W(s, t) d t d s \\
& +\int_{0}^{x} D_{1} G(\sqrt{W(s, 0)}) d s \\
& =\frac{1}{2} \int_{0}^{x}\left[\left.\frac{D_{1} f(s, t)}{g(\sqrt{W(s, t)}) \sqrt{W(s, t)}}\right|_{0} ^{y}\right] d s \\
& -\frac{1}{2} \int_{0}^{x} \int_{0}^{y} D_{1} f(s, t) \frac{-1}{g^{2}(\sqrt{W(s, t)}) W(s, t)}\left[g^{\prime}(\sqrt{W(s, t)}) \frac{1}{2} \frac{D_{2} W(s, t)}{(\sqrt{W(s, t)})}(\sqrt{W(s, t)})\right. \\
& \left.+g(\sqrt{W(s, t)}) \frac{1}{2} \frac{D_{2} W(s, t)}{(\sqrt{W(s, t)})}\right] d t d s+\frac{1}{2} \int_{0}^{x} \int_{0}^{y} B(s, t) d t d s \\
& -\frac{1}{4} \int_{0}^{x} \int_{0}^{y} \frac{g^{\prime}(\sqrt{W(s, t)}) \sqrt{W(s, t)}+g(\sqrt{W(s, t)})}{g^{2}(\sqrt{W(s, t)})(W(s, t))^{3 / 2}} D_{1} W(s, t) D_{2} W(s, t) d t d s \\
& +\int_{0}^{x} D_{1} G(\sqrt{W(s, 0)}) d s \\
& =\frac{1}{2} \int_{0}^{x} \frac{D_{1} f(s, y)}{g(\sqrt{W(s, y)}) \sqrt{W(s, y)}} d s-\frac{1}{2} \int_{0}^{x} \frac{D_{1} f(s, 0)}{g(\sqrt{W(s, 0)}) \sqrt{W(s, 0)}} d s
\end{aligned}
$$




$$
\begin{aligned}
& +\frac{1}{4} \int_{0}^{x} \int_{0}^{y} \frac{g^{\prime}(\sqrt{W(s, t)}) \sqrt{W(s, t)}+g(\sqrt{W(s, t)})}{g^{2}(\sqrt{W(s, t)})(W(s, t))^{3 / 2}} D_{2} W(s, t)\left(D_{1} f(s, t)-D_{1} W(s, t)\right) d t d s \\
& +\frac{1}{2} \int_{0}^{x} \int_{0}^{y} B(s, t) d t d s+\int_{0}^{x} D_{1} G(\sqrt{W(s, 0)}) d s \\
\leq & \frac{1}{2} \int_{0}^{x} \frac{D_{1} f(s, y)}{g(\sqrt{f(s, y)}) \sqrt{f(s, y)}} d s-\frac{1}{2} \int_{0}^{x} \frac{D_{1} W(s, 0)}{g(\sqrt{W(s, 0)}) \sqrt{W(s, 0)}} d s \\
& +\frac{1}{2} \int_{0}^{x} \int_{0}^{y} B(s, t) d t d s+\int_{0}^{x} D_{1} G(\sqrt{W(s, 0)}) d s \\
= & \left.G \sqrt{f(s, y)}\right|_{0} ^{x}-\int_{0}^{x} D_{1} G(\sqrt{W(s, 0)}) d s+\frac{1}{2} \int_{0}^{x} \int_{0}^{y} B(s, t) d t d s+\int_{0}^{x} D_{1} G(\sqrt{W(s, 0)}) d s \\
= & G(\sqrt{f(x, y)})-G(\sqrt{f(0, y)})+\frac{1}{2} \int_{0}^{x} \int_{0}^{y} B(s, t) d t d s \\
= & G(\sqrt{f(x, y)})-G(\sqrt{W(0, y)})+\frac{1}{2} \int_{0}^{x} \int_{0}^{y} B(s, t) d t d s .
\end{aligned}
$$

which implies

$$
G(\sqrt{W(x, y)}) \leq G(\sqrt{f(x, y)})+\frac{1}{2} \int_{0}^{x} \int_{0}^{y} B(s, t) d t d s .
$$

Since $G^{-1}$ is increasing, from $(2.9)$ we have

$$
\sqrt{W(x, y)} \leq G^{-1}\left[G(\sqrt{f(x, y)})+\frac{1}{2} \int_{0}^{x} \int_{0}^{y} B(s, t) d t d s\right]
$$

Using (2.10) in $U(x, y) \leq \sqrt{W(x, y)}$, we get the required inequality in (2.5).

Remark 2. We note that Theorem B is a special case of Theorem 2.

\section{Applications}

Consider the nonlinear integral equation of the form

$$
X^{2}(t)=j(t)+\int_{0}^{t} J(t, s, X(s)) d s .
$$

where $j(t): R_{+} \rightarrow R, J: D \times R \rightarrow R$ are continouous functions. For the existence and other properties of the solutions of the slight variants of equation (3.1), see [2,3] and some of the references cited therein.

We assume that the functions $j, J$ in (3.1) satisfy the conditions

$$
|j(t)| \leq f(t), \quad|J(t, s, X(s))| \leq K(t, s)|X(s)|
$$




$$
Q(t)=\sqrt{f(t)}+\frac{1}{2} \int_{0}^{t} A(s) d s<\infty
$$

where $f(t), K(t, s)$ and $A(t)$ are as in Theorem 1 .

Let $X(t), t \in R_{+}$be a solution of (3.1). From (3.1) and (3.2) we have

$$
|X(t)|^{2} \leq f(t)+\int_{0}^{t} K(t, s)|X(s)| d s .
$$

An application of the inequality in $\left(c_{1}\right)$ given in Theorem 1 to (3.4) yields

$$
|X(t)| \leq Q(t),
$$

for $t \in R_{+}$. From the hypothesis (3.3), the estimation in (3.5) implies the boundedness of the solution $X(t)$ of $(3.1)$ on $R_{+}$.

We now consider the equation (3.1) under the following conditions on the functions $j, J$ in (3.1):

$$
|j(t)| \leq f(t) e^{-\alpha t}, \quad|J(t, s, X(s))| \leq K(t, s) e^{-\alpha\left(t-\frac{1}{2} s\right)}|X(s)|,
$$

where $\alpha \geq 0$ is a constant and hypothesis (3.3) is satisfied.

Let $X(t)$ be a solution of (3.1). Then from (3.1), (3.6) we have

$$
|X(t)|^{2} \leq f(t) e^{-\alpha t}+\int_{0}^{t} K(t, s) e^{-\alpha\left(t-\frac{1}{2} s\right)}|X(s)| d s
$$

From (3.7) we observe that

$$
\left(|X(t)| e^{\frac{1}{2} \alpha t}\right)^{2} \leq f(t)+\int_{0}^{t} K(t, s)\left(|X(s)| e^{\frac{1}{2} \alpha s}\right) d s .
$$

Now applying the inequality in $\left(c_{1}\right)$ given in Theorem 1 to (3.8), and then multiplying the resulting inequality by $e^{-\frac{1}{2} \alpha t}$, we obtain

$$
|X(t)| \leq Q(t) e^{-\frac{1}{2} \alpha t}
$$

for $t \in R_{+}$. From (3.3) and (3.9) we see that the solution $X(t)$ of equation (3.1) approach 0 as $t \rightarrow \infty$.

\section{References}

[1] D. Bainov and P. Simenov, Integral Inequalities and Applications, Kluwer Academic Publishers, Dordrecht, 1992.

[2] W. Okrasinski, On a nonlinear convolution equation occurring in the theory of water precloation, Ann. Polon. Math. 37(1980), 223-229.

[3] B. G. Pachpatte, Inequalities for Differential and Integral Equatons, Academic Press, New York, 1998. 
[4] B. G. Pachpatte, Inequalities for Finite Difference Equations, Mrcel Dekker Inc., New York, 2001.

[5] B. G. Pachatte, Explicit bounds on Volterra type integral inequalities. Tamsui Oxford J. Math. Sci. 19(2003), 13-25.

Center for General Education, Northern Taiwan Institute of Science and Technology, Peito, Taipei, Taiwan 11202.

Department of Mathematics, Tamkang University, Tamsui, Taiwan 25137. 